\title{
Legume consumption and risk of hypertension in a prospective cohort of Chinese men and women
}

\author{
Fang Guo ${ }^{1,2,3}$, Qiang Zhang, ${ }^{3,4}$, Yue Yin ${ }^{3 *}$, Yan Liu $^{3}$, Hong Jiang 3 , Ni Yan ${ }^{3}$, Jing Lin $^{3}$, \\ Xiao-hong $\mathrm{Liu}^{1 *}$ and Le $\mathrm{Ma}^{3,5 *}$ \\ ${ }^{1}$ The First Affiliated Hospital, Xi'an Jiaotong University Health Science Center, Xi'an 710061, People's Republic of China \\ ${ }^{2}$ School of Public Health, The University of Hong Kong, Pok Fu Lam, Hong Kong Special Administrative Region 999077, People's \\ Republic of China \\ ${ }^{3}$ School of Public Health, Xi'an Jiaotong University Health Science Center, Xi'an 710061, People's Republic of China \\ ${ }^{4}$ Department of Biomedical Engineering, the Hong Kong Polytechnic University, Hung Hom, Hong Kong 999077, People's \\ Republic of China \\ ${ }^{5}$ Key Laboratory of Environment and Genes Related to Diseases, Xi'an Jiaotong University, Ministry of Education of China, Xi'an \\ 710061, People's Republic of China
}

(Submitted 20 January 2019 - Final revision received 21 October 2019 - Accepted 25 October 2019 - First published online 7 November 2019)

\begin{abstract}
Prior data on long-term association between legume consumption and hypertension risk are sparse. We aimed to evaluate whether total legume and subtype intakes prospectively associate with hypertension incidence among 8758 participants ( $\geq 30$ years) from the China Health and Nutrition Survey 2004-2011. Diet was assessed by interviews combining 3-d 24-h food recalls and household food inventory weighing at each survey round. Incident hypertension was identified by self-reports or blood pressure measurements. We applied multivariable Cox regressions to estimate hazard ratios (HR) with corresponding $95 \% \mathrm{CI}$ for hypertension across increasing categories of cumulatively averaged legume intakes. For 35990 person-years (median 6.0 years apiece), we documented 944 hypertension cases. After covariate adjustment, higher total legume intakes were significantly associated with lower hypertension risks, with HR comparing extreme categories being 0.56 (95\% CI 0.43 , $0.71 ; P_{\text {trend }}<0.001$ ). Then we found that intakes of dried legumes (HR 0.53 (95\% CI 0.43, 0.65); $P_{\text {trend }}<0.001$ ) and fresh legumes (HR 0.67 (95\% CI $\left.0.55,0.81) ; P_{\text {trend }}<0.001\right)$ were both related to decreased hypertension hazards. However, further dried legume classification revealed that negative association with hypertension substantially held for soyabean (HR 0.51 (95\% CI 0.41, 0.62); $P_{\text {trend }}<0.001$ ) but not non-soyabean intakes. In stratified analyses, the association of interest remained similar within strata by sex, BMI, physical activity, smoking and drinking status; rather, significant heterogeneity showed across age strata $\left(P_{\text {interaction }}=0 \cdot 02\right)$. Total legume consumption among the over-65s was related to a more markedly reduced hypertension risk (HR 0.47 (95\% CI 0.30, 0.73); $\left.P_{\text {trend }}<0.001\right)$. Our findings suggest an inverse association of all kinds of legume (except non-soyabean) intakes with hypertension risks.
\end{abstract}

Key words: Legumes: Soyabeans: Hypertension: Blood pressure: Chinese adults: Prospective studies

Hypertension poses a massive public health and economic burden globally because of its high incidence rate and resulting CVD, chronic kidney disease and premature mortality ${ }^{(1)}$. It is estimated that one third of the world population over 20 years of age, approximately 1.4 billion people, were suffering from hypertension $^{(2)}$. In China, with rapid urbanisation and ageing of the population, the prevalence of hypertension has increased significantly in recent years, reaching $44.7 \%$ among Chinese adults aged $35-75$ years in $2017^{(3)}$. Hence, it is of great importance to ascertain modifiable risk factors to prevent the onset of this disease.

Since the Dietary Approaches to Stop Hypertension trial elucidated that diet rich in fruits, vegetables and low-fat dairy foods could reduce blood pressure (BP), the nutritional strategy as an effective approach to reduce the risk of hypertension has aroused an increasing scientific interest ${ }^{(4,5)}$. Legumes/beans, an indispensable part of plant-based diet, are packed with a variety of beneficial nutrients including dietary fibre, vegetable protein

Abbreviations: BP, blood pressure; CHNS, China Health and Nutrition Survey; EER, estimated energy requirement; EI, energy intake; HR, hazard ratio.

*Corresponding authors: Le Ma, fax +86 029 82657475, email male@mail.xitu.edu.cn; Yue Yin, email lycwr@hotmail.com; Xiao-hong Liu, email liuxiaoh@mail. xjtu.edu.cn 
and minerals such as $\mathrm{K}$, which are associated with the reduction of $\mathrm{BP}^{(6)}$. Laboratory studies also suggested that legume intake may decrease in vivo oxidation, reduce systemic inflammation and improve arterial compliance, thereby showing a potential protective role against hypertension incidence ${ }^{(7)}$. Up to now, several retrospective studies and small-scale randomised clinical trials have examined the effects of various legume intakes on BP, but the conclusions were inconsistent ${ }^{(8-14)}$. Most randomised clinical trials probed the potential advantages of legumes supplemented for a short period at a relatively high dose among overweight/obese group ${ }^{(9,10)}$ or patients with the metabolic syndrome $^{(11)}$ or diabetes ${ }^{(12,13)}$. The present available evidences have limited power to detect the long-term association between habitual legume consumption and incident hypertension in general population. Thus, large-scale prospective studies in support of this plausible association are admittedly needed. However, to our knowledge, only a few prospective studies have investigated the associations of legumes or specific subtypes with BP change or hypertension and the results remained highly conflicting ${ }^{(15-17)}$. Further, different types of legumes may differ in their nutritional contents and have discrepant impacts on risk of hypertension ${ }^{(18,19)}$.

As an important part of Chinese traditional diet since ancient times, more than twenty legume species are grown and about 200 kinds of legume foodstuffs (e.g. soyabean, adzuki bean, common bean, cowpea, bean sprout, etc.) are consumed in China $^{(20)}$. The average intake of legumes such as soyabeans was reported to be $>100 \mathrm{~g} / \mathrm{d}$ among Chinese population, which is nearly three times that among European and American populations ${ }^{(21)}$. In this context, we therefore utilised data from the China Health and Nutrition Survey (CHNS) to evaluate the longitudinal relationship between habitual legume intakes and subsequent incidence of hypertension in the general Chinese population.

\section{Materials and methods}

\section{Study population}

Candidates in the present study were participants in the CHNS, which is a nationwide survey on health risk factors, nutritional status and health-related outcomes of its population. The CHNS is an ongoing open-cohort study conducted since 1989, with the data followed up and released every 2-4 years. A multistage, random cluster sampling method was utilised to draw the individuals surveyed from twelve diverse provinces across China. Details of the cohort and sampling procedure have been published elsewhere ${ }^{(22)}$. To investigate the associations of dietary legumes with hypertension, we used data from CHNS survey cycles from 2004, 2006, 2009 to 2011. The reasons for choosing these waves were mainly concerning the changes in dietary information collection methods in 2004. Specifically, all foods and condiments were measured using Chinese balance scales (graduation: $10 \mathrm{~g}$ ), recorded with traditional Chinese mass unit (JIN) and further coded based on 1991 China Food Composition Tables in surveys before 2004. By contrast, digital kitchen scales (graduation: $1 \mathrm{~g}$ ) with international mass unit $(\mathrm{g})$ and updated version of Food Composition Tables (2002/2004) were adopted in the following rounds $(2004-2011)^{(23,24)}$. To maintain consistency and reduce measurement bias, we herein did not cover the records before 2004 .

The participants were restricted to those aged $\geq 30$ years, ensuring a relatively high incidence of hypertension. We then excluded pregnant women during the follow-up period. From the 15818 candidates, those with an indistinct or missing diagnosis of hypertension over the four waves of survey were accordingly ruled out. After eliminating hypertensives at baseline survey, 13851 subjects free of hypertension were potentially eligible for our cohort study. Individuals were also removed if their 24-h dietary recall was uncompleted with missing information on any survey day, or total energy intake (EI) was identified as unusual (outside the range of 3.34-25.12 MJ/d for men and $2.51-16.74 \mathrm{MJ} / \mathrm{d}$ for women $)^{(25)}$. Among the remaining subjects, 4878 were excluded due to loss to follow-up after baseline ( $n$ 1751) or first entrance into the CHNS in 2011 ( $n$ 3127). Finally, there left a total of 8758 participants for the analysis (Fig. 1). The present study was reviewed and approved by Institutional Review Boards of the University of North Carolina, Chapel Hill and the Chinese Centre for Disease Control. Each subject gave written informed consent for participation prior to the surveys.

\section{Exposure measurement}

In each CHNS cycle, data of dietary intakes were collected via three consecutive 24-h dietary recalls at the individual level, in

Participants in CHNS 2004-2011 (n 23 283)

Excluded if participants aged $<30$ years

(n 7362) or got pregnant ( $n$ 103)

Participants aged $\geq 30$ years without pregnant women ( $n$ 15 818)

Excluded if data on diagnosis of

hypertension were unavailable $(n 7)$

Participants with intact information on hypertension diagnosis (n 15811$)$

Excluded if participants were diagnosed with hypertension before baseline ( $n$ 1960)

Participants free from hypertension at baseline ( $n$ 13 851)

Excluded if data on 24-h dietary recall were missing ( $n 153)$ or energy intake was implausible ( $n$ 62)

Eligible participants ( $n 13636$ )

Participants lost to follow-up ( $n$ 1751) or entered into CHNS in 2011 ( $n$ 3127)

Baseline sample for analysis ( $n$ 8758)

Fig. 1. Flow chart of sample selection from the China Health and Nutrition Survey (CHNS). 
combination with conversion of cooking ingredients (such as oils and condiments) at the household level acquired by the food inventory weighing method. Participants' food intakes were thus updated at every follow-up survey, and the median time of dietary assessments was three. Dietary investigation was performed by trained staff using a standardised protocol, of which the accuracy was validated previously against the doubly labelled water method for energy (men: $r$ 0.56; women: $r 0 \cdot 60)^{(26)}$ and the 3-d dietary recalls were highly correlated with household food inventory weights for food groups (e.g. $r 0.76$ for legume $)^{(27)}$. We calculated the estimated energy requirement (EER) for each subject with the use of total energy expenditure equations published in the US/Canadian Dietary Reference Intake report for normal-weight and overweight adults, in which sex, age, weight, height and physical activity level are accounted for using a doubly labelled water database ${ }^{(28)}$. Energy misreporting was evaluated by calculating the ratio of reported EI to EER with an expected value of $1 \cdot 0$. Its $95 \%$ confidence limits with amount of variation inherent in EI and EER data considered were computed as cut-off values to define misreporters ${ }^{(29)}$. Specifically, the calculation was realised by the formula $95 \%$ confidence limit $= \pm 2 \times \sqrt{\left(\mathrm{CV}_{\mathrm{wEI}}^{2} / \mathrm{d}\right)+\left(\mathrm{CV}_{\mathrm{wEER}}\right)^{2}}$, where $d$ was the number of days of diet assessment (in this case, 3 ). $\mathrm{CV}_{\mathrm{wEI}}$ was the $\mathrm{CV}$ for $\mathrm{EI}$ records and assumed to have a pooled average value of $23 \%{ }^{(30)}$. $\mathrm{CV}_{\text {wEER }}$ represented the $\mathrm{CV}$ for EER and was taken as $8 \cdot 2 \%^{(29)}$. By this approach, subjects were defined as acceptable, under- or over-reporters of EI as having a ratio of EI:EER in the range $0.69-1 \cdot 31,<0.69$ or $>1 \cdot 31$, respectively.

In the present study, the total legume consumption was estimated as the sum of dried legumes and fresh legumes. According to the Chinese Food Composition Tables, dried legumes could be further classified into two types due to discrepant nutrient compositions, one is soyabean (e.g. soyabean, black soyabean, tofu and soyamilk) and the other is non-soyabean (e.g. mung bean, adzuki bean, red kidney bean and pinto bean). Comparatively, fresh legumes were defined including common bean, bean sprout, string bean, cowpea, etc. Nutrient intakes were calculated as multiplying the ingestion of each food item by the nutrient content of a standard portion size of $100 \mathrm{~g}$ based on the Food Composition Tables. We used nutrient density models to obtain energy-adjusted intakes $(\mathrm{g} / 1000 \mathrm{kcal}(4184 \mathrm{~kJ}))$ instead of crude intakes, to account for confounding of total energy requirements regarding body size, physical activity and metabolic efficiency ${ }^{(31)}$. Given the large proportion of subjects who did not report consumption of legumes and especially certain subtypes during 3 -d probe period, the total legume and each subtype intakes were categorised by quartiles in addition to non-consumption, with the non-consumers set as the reference group.

\section{Outcome measurement}

The outcome of the present study was incident hypertension that occurred during the follow-up. At the baseline and each round of follow-up survey, hypertension was defined as a self-report of diagnosis by a physician, through the question 'Has a doctor ever told you that you suffer from high blood pressure?' For those who did not answer this question or answered 'no' or 'unknown', we further examined their three-time averaged BP values. Consistent with the WHO conventions (1999), hypertension was diagnosed as average systolic BP/diastolic BP $\geq 140 / 90 \mathrm{mmHg}$. The calendar year of diagnosis was recorded to estimate a timeto-event endpoint with the view of survival analysis. Regularly calibrated mercury sphygmomanometers with appropriate-sized cuffs were applied to measure BP by trained examiners. Measurements were taken in triplicate after at least a 10-min rest, and the three readings were averaged as the corresponding $\mathrm{BP}$ values to reduce measurement errors.

\section{Assessments of covariates}

Specific information of participants on demographic, socioeconomic, lifestyle and dietary factors was collected and processed by certified health professionals via questionnaire-based interviews. Educational attainment was categorised into three levels (6 years/7-9 years/ $\geq 10$ years). We separated inflationadjusted per capita household income into tertiles (low, medium and high). The urbanicity index, a multidimensional metrics of urbanisation, was divided into tertiles. Tobacco and alcohol consumption were both dichotomised as never or ever. Physical activity covered four domains in the CHNS: occupation, household, leisure time and transportation. Time spent in each activity was multiplied by its assigned metabolic equivalent intensity in accordance with the Compendium of Physical Activities ${ }^{(32)}$. The total metabolic equivalents were then trisected as light, moderate and vigorous efforts. Height was scaled with the subject barefoot to the nearest $0 \cdot 1 \mathrm{~cm}$, and weight was estimated to the nearest $0 \cdot 1 \mathrm{~kg}$ with light clothing. BMI was computed as weight in $\mathrm{kg}$ divided by squared height in $\mathrm{m}$. We also included other dietary covariates, such as total dietary energy, total fruits and vegetables and total red meat intake, as the surrogates to control for potential confounding from dietary pattern.

\section{Statistical analysis}

We termed the first available measurement as baseline and deemed events to occur in the midpoint between wave of the first hypertension diagnosis and the previous wave because of the insidious onset of hypertension. To minimise withinperson variation and thus generate better estimates of long-term intake, a cumulative average intake value was calculated and utilised. Cox proportional hazards assessed associations between legume intake and hypertension through 2011, with time at risk from a baseline questionnaire collected until first event, death or censoring at return of the most recent valid record, whichever came first. The proportional hazards assumption for legume intakes and other covariates was checked by Schoenfeld's residuals, and no violation was found $(P>0.05)$. Hazard ratios (HR) were applied to measure relative risks of hypertension in higher intake categories compared with the non-consumption group, and $95 \% \mathrm{CI}$ were calculated. The following sequential models were built to account for potential confounders in the aforementioned analyses: model 1 presented the age and sex-adjusted results; model 2 added baseline sociodemographic factors (such as education, income and urbanised index) and lifestyle factors (including physical activity, smoking status, alcohol intake, BMI and past history of diabetes mellitus) 
into multivariable analysis; model 3 additionally adjusted for total EI and potential dietary confounders, namely total fruit and vegetable, and total red meat intakes.

To evaluate confounding and effect modifications by demographic and lifestyle factors, we conducted stratified analyses by sex (male and female), age at baseline ( $<65$ and $\geq 65$ years), BMI $\left(<24\right.$ and $\geq 24 \mathrm{~kg} / \mathrm{m}^{2}$ ), physical activity ( $<$ median level and $\geq$ median level), smoking (never and ever) and drinking (never and ever) status. Tests for interaction were performed by including a product term between each stratifying variable and total legume intake in the full model and evaluating Wald $P$ values. In addition, we verified robustness of the primary results via conducting several sensitivity analyses: (1) recalculate the personyears by assuming the last time of visit as the date of incident hypertension or quit, not using the middle point between follow-up intervals; (2) further adjust for baseline systolic BP and diastolic BP; (3) further account for important confounding effect of salt consumption by including $\mathrm{Na}$ intake as a surrogate in the final model; (4) since a history of diabetes, stroke or myocardial infarction was, on average, highly related to hypertension development and drastic change in dietary habits after perceiving these conditions, we repeated the analysis excluding adults with indications of these diseases at baseline. Tests for trend were performed by means of entering the categories as continuous variables in each model. Considering multiple dietary factors often show high correlation with one another, we estimated the effect of collinearity within them. All dietary variables added into the model had a variable inflation factor value $<2$ (mean $1.03($ SD 0.04$))^{(33)}$, and their correlation coefficients were less than 0.3 (mean $0 \cdot 10$ (SD 0.09)). All analyses were completed with SPSS version 13.0 for Windows (IBM SPSS Inc.). All $P$-values were two-sided, and statistical significance was set at $P \leq 0.05$.

\section{Results}

During 35990 person-years (6.0 median years) of follow-up, a total of 944 cases of hypertension were identified, with the incidence rate being approximately $10 \cdot 8 \%$. The baseline characteristics of the study population by intake of total legumes are shown in Table 1. Men and women in the higher categories of total legume intake were slightly older and more physically active with somewhat higher diastolic BP levels. A higher legume intake was correlated with decreased consumption of alcohol, higher individual income and higher degree of urbanicity. With respect to dietary factors, those who consumed more total legumes were, in general, more likely to have higher intake of fruits and vegetables, but lower intake of total red meats and total energy intake. As for energy reporting status, the higher the legume intake category, the lower the energy misreporting rate. Overall, the proportion of reporting acceptable EI was relatively high as approximately $73.3 \%$. We also compared the demographic and health-related factors between the withdrawn and included subjects (online Supplementary Table S1). Those who were lost to follow-up tended to be slightly younger with lighter physical activity levels. Also, they were more likely to gain higher education and have higher income and urbanisation index. While minor differences were observed in intakes of total energy, fruit and vegetables as well as red meat between these two groups, the difference in total legume intake was not statistically significant.

Table 2 gives the associations between consumption of various legume types and incident hypertension. In the ageand sex-adjusted model, total legume intake was modestly associated with reduced hypertension risks; a significant effect estimate was observed for the highest intake group (HR 0.64; $95 \%$ CI $0.50,0.82 ; P_{\text {for trend }}=0.01$ ) compared with the nonconsumption group. Additional control for non-dietary risk factors slightly strengthened our results and the trend test yielded a $P$ value smaller than 0.001. After further adjustment for dietary factors, the inverse association between total legume intake and hypertension hazard still held, with HR and 95\% CI for higher categories being 0.70 (95\% CI 0.55, 0.90), 0.48 (95\% CI 0.37 , $0.62), 0.53(95 \%$ CI $0.41,0.67)$ and 0.56 (95\% CI $0.43,0.71)$ $\left(P_{\text {for trend }}<0.001\right)$.

In terms of the specific associations for individual types of legumes, participants showed a $47 \%$ (95\% CI 0.43, 0.65; $\left.P_{\text {for trend }}<0.001\right)$ lower chance of developing hypertension from a higher intake of dried legumes in the top category when compared with non-consumers. Similarly, greater fresh legume intakes were also significantly associated with lower hypertension risks (HR 0.67; $95 \%$ CI $0.55,0.81$ ) by using the same fullmodel adjustment. When dried legumes were further classified into soyabeans and non-soyabeans, the significant inverse association with hypertension was only shown for soyabeans, with fully adjusted HR comparing extreme categories as 0.51 (95\% CI 0.41, 0.62; $\left.P_{\text {for trend }}<0.001\right)$. Regarding non-soyabean consumption, the HR of hypertension in the final multivariate model was non-significantly lower in the top intake group (HR 0.88; $95 \%$ CI 0.65, 1.20).

In the a priori-determined stratified analysis (Table 3), the inverse association of total legume intake with the development of hypertension was mostly unchanged among participants with various risk profiles characterised by sex, BMI, physical activity, smoking and drinking status ( $\left.P_{\text {for interaction }}>0 \cdot 05\right)$. However, results appeared to distinguish across the age strata, with significant interaction of legume consumption with baseline age detected $\left(P_{\text {for interaction }}=0 \cdot 02\right)$. The multivariate HR of hypertension comparing top category of total legume intake with nonconsumption was 0.47 (95\% CI $\left.0.30,0.73 ; P_{\text {for trend }}<0.001\right)$ among the elderly ( $\geq 65$ years). In contrast, the association of interest was less pronounced but still significant in the nonelderly subgroup (HR 0.61; $95 \%$ CI $0.45,0.83 ; P_{\text {for trend }}=0.04$ ).

To examine the robustness of these observed associations, we conducted several sensitivity analyses (online Supplementary Table S2). Similar results were derived when we recalculated the person-years not using the middle point between follow-up intervals. Further adjustment for baseline systolic BP and diastolic BP or the potential confounding effect of $\mathrm{Na}$ intake in the final model yielded nearly the same findings. When we repeated the analyses after excluding adults with indications of chronic metabolic diseases at baseline, the inverse association was materially unchanged with the HR for hypertension in the highest total legume intake group being 0.53 (95\% CI 0.41, 0.69). 
Table 1. Baseline characteristics of participants aged $\geq 30$ years in China Health and Nutrition Survey (2004-2011) according to total legume intake (Mean values and standard deviations; percentages)

\begin{tabular}{|c|c|c|c|c|c|c|c|c|c|c|c|c|c|c|c|c|}
\hline \multirow[b]{3}{*}{ Characteristics* $^{*}$} & \multicolumn{16}{|c|}{ Total legume intake } \\
\hline & \multicolumn{3}{|c|}{ Non-consumers } & \multicolumn{3}{|c|}{ Q1 (lower) } & \multicolumn{3}{|c|}{ Q2 } & \multicolumn{3}{|c|}{ Q3 } & \multicolumn{3}{|c|}{ Q4 (higher) } & \multirow[b]{2}{*}{$P \dagger$} \\
\hline & Mean & & SD & Mean & & SD & Mean & & SD & Mean & & SD & Mean & & SD & \\
\hline No. of subjects & & 749 & & & 2002 & & & 2002 & & & 2003 & & & 2002 & & \\
\hline \multicolumn{17}{|c|}{ Legume intake (g/1000 kcal (4184 kJ)) } \\
\hline Median & & 0.0 & & & $20 \cdot 7$ & & & $39 \cdot 1$ & & & $60 \cdot 3$ & & & 97.4 & & \\
\hline Range & & & & & $<30.0$ & & & $30 \cdot 0,49 \cdot 0$ & & & $49 \cdot 0,74 \cdot 3$ & & & $\geq 74.3$ & & \\
\hline Age at cohort entry (years) & 51.8 & & 14.4 & $48 \cdot 7$ & & $12 \cdot 7$ & $49 \cdot 3$ & & $12 \cdot 6$ & $49 \cdot 2$ & & $12 \cdot 2$ & $49 \cdot 6$ & & $12 \cdot 8$ & $<0.001$ \\
\hline Male (\%) & & $51 \cdot 1$ & & & 50.9 & & & 49.5 & & & $48 \cdot 0$ & & & $45 \cdot 4$ & & 0.004 \\
\hline $\mathrm{BMI}\left(\mathrm{kg} / \mathrm{m}^{2}\right)$ & $22 \cdot 9$ & & $3 \cdot 3$ & $23 \cdot 0$ & & $3 \cdot 1$ & $23 \cdot 1$ & & $3 \cdot 2$ & $23 \cdot 3$ & & $3 \cdot 2$ & $23 \cdot 3$ & & $3 \cdot 2$ & $<0.001$ \\
\hline \multicolumn{17}{|l|}{ Physical activity level (\%)§ } \\
\hline Light & & 41.1 & & & $35 \cdot 1$ & & & 33.3 & & & $30 \cdot 8$ & & & $31 \cdot 2$ & & $<0.001$ \\
\hline Moderate & & 27.5 & & & $32 \cdot 0$ & & & 33.9 & & & 34.6 & & & $35 \cdot 0$ & & \\
\hline Vigorous & & 31.4 & & & $32 \cdot 9$ & & & $32 \cdot 8$ & & & 34.6 & & & 33.8 & & \\
\hline Cigarette smoker (\%) & & 33.0 & & & $35 \cdot 3$ & & & $35 \cdot 0$ & & & 34.3 & & & $32 \cdot 7$ & & 0.38 \\
\hline Alcohol consumption (\%) & & $30 \cdot 6$ & & & $36 \cdot 5$ & & & $36 \cdot 0$ & & & $35 \cdot 0$ & & & $32 \cdot 4$ & & 0.005 \\
\hline \multicolumn{17}{|l|}{ Education attained $(\%)$} \\
\hline $0-6$ years & & $46 \cdot 3$ & & & $42 \cdot 9$ & & & 43.7 & & & 44.4 & & & 43.7 & & 0.68 \\
\hline $7-9$ years & & 33.1 & & & $32 \cdot 9$ & & & 33.1 & & & 31.8 & & & 32.5 & & \\
\hline$\geq 10$ years & & $20 \cdot 6$ & & & $24 \cdot 2$ & & & 23.2 & & & 23.8 & & & $23 \cdot 8$ & & \\
\hline \multicolumn{17}{|l|}{ Income level (\%) } \\
\hline Low & & $37 \cdot 0$ & & & $33 \cdot 5$ & & & 33.0 & & & $32 \cdot 4$ & & & $31 \cdot 7$ & & 0.008 \\
\hline Median & & $35 \cdot 1$ & & & $35 \cdot 2$ & & & $34 \cdot 3$ & & & $33 \cdot 3$ & & & $32 \cdot 7$ & & \\
\hline High & & 27.9 & & & 31.3 & & & $32 \cdot 7$ & & & 34.3 & & & $35 \cdot 7$ & & \\
\hline \multicolumn{17}{|l|}{ Urbanisation index level (\%) } \\
\hline Low & & $39 \cdot 3$ & & & 34.8 & & & 33.7 & & & $30 \cdot 4$ & & & $32 \cdot 3$ & & $<0.001$ \\
\hline Median & & 24.7 & & & $32 \cdot 2$ & & & 32.5 & & & $36 \cdot 1$ & & & $36 \cdot 9$ & & \\
\hline High & & $36 \cdot 0$ & & & 33.0 & & & 33.8 & & & 33.5 & & & $30 \cdot 8$ & & \\
\hline $\begin{array}{l}\text { Total fruits and vegetables } \\
(\mathrm{g} / 1000 \mathrm{kcal}(4184 \mathrm{~kJ}))\end{array}$ & $182 \cdot 9$ & & $103 \cdot 1$ & $162 \cdot 4$ & & $74 \cdot 6$ & $178 \cdot 3$ & & 78.9 & $192 \cdot 8$ & & $86 \cdot 0$ & $220 \cdot 8$ & & $110 \cdot 1$ & $<0.001$ \\
\hline $\begin{array}{l}\text { Total red meat } \\
(\mathrm{g} / 1000 \mathrm{kcal}(4184 \mathrm{~kJ}))\end{array}$ & $34 \cdot 2$ & & $31 \cdot 8$ & $40 \cdot 0$ & & $27 \cdot 5$ & $38 \cdot 3$ & & $26 \cdot 3$ & $35 \cdot 8$ & & $26 \cdot 2$ & $32 \cdot 2$ & & $24 \cdot 9$ & $<0.001$ \\
\hline Total energy $(k c a l \ddagger / d)$ & $2085 \cdot 8$ & & 655.0 & $2336 \cdot 0$ & & 636.6 & 2287.8 & & 581.1 & 2241.9 & & 574.7 & $2092 \cdot 3$ & & 534.4 & $<0.001$ \\
\hline SBP (mmHg) & $122 \cdot 0$ & & $18 \cdot 3$ & $120 \cdot 6$ & & $16 \cdot 7$ & $120 \cdot 4$ & & $16 \cdot 2$ & $120 \cdot 0$ & & $15 \cdot 9$ & 120.9 & & $16 \cdot 3$ & 0.10 \\
\hline $\mathrm{DBP}(\mathrm{mmHg})$ & 78.6 & & $10 \cdot 8$ & $77 \cdot 8$ & & $10 \cdot 6$ & $78 \cdot 0$ & & $10 \cdot 2$ & $78 \cdot 0$ & & $10 \cdot 2$ & $79 \cdot 0$ & & $10 \cdot 3$ & 0.002 \\
\hline El:EER ratio & 1.03 & & 0.33 & $1 \cdot 13$ & & 0.32 & $1 \cdot 10$ & & 0.29 & 1.07 & & 0.29 & 1.01 & & 0.29 & $<0.001$ \\
\hline \multicolumn{17}{|l|}{ Energy reporting status (\%)\| } \\
\hline Under-reporting & & $12 \cdot 4$ & & & 4.7 & & & $5 \cdot 3$ & & & $6 \cdot 7$ & & & $10 \cdot 0$ & & $<0.001$ \\
\hline Acceptable reporting & & $70 \cdot 3$ & & & 71.2 & & & 73.0 & & & 74.6 & & & 75.5 & & \\
\hline Over-reporting & & $17 \cdot 3$ & & & $24 \cdot 1$ & & & $21 \cdot 7$ & & & $18 \cdot 7$ & & & 14.5 & & \\
\hline
\end{tabular}

$\mathrm{Q}$, quartile; SBP, systolic blood pressure; DBP, diastolic blood pressure; EI, reported energy intake; EER, estimated energy requirement.

* Data are expressed as means and standard deviations for continuous variables, or percentages for categorical variables.

t Derived from tests of discrepancies across all categories of total legume intake, by ANOVA, Kruskal-Wallis test or $\chi^{2}$ test as appropriate.

$\ddagger$ To convert kcal to $\mathrm{kJ}$, multiply by 4.184 .

$\S$ Energy expenditure on total physical activity; MET-h/week (metabolic equivalent task hours for each week) was calculated by multiplying the amount of time spent in each activity with the average MET values of each category

II The energy reporting status is determined by $95 \%$ confidence limits of ratio of El to EER. 
Table 2. Risk of incident hypertension by categories of cumulative average intake of total legumes and subtypes in the China Nutrition and Health Study (CHNS) 2004-2011 (Hazard ratios (HR) and $95 \%$ confidence intervals)

\begin{tabular}{|c|c|c|c|c|c|c|c|c|c|c|c|}
\hline & \multicolumn{10}{|c|}{ Levels of total legume and its subtype intake } & \multirow{3}{*}{$\begin{array}{c}P_{\text {for }} \\
\text { trend }\end{array}$} \\
\hline & \multirow{2}{*}{$\begin{array}{c}\text { Non-consumers } \\
\left(\text { ref) }{ }^{*}\right.\end{array}$} & \multicolumn{3}{|c|}{ Q1 } & \multicolumn{2}{|c|}{ Q2 } & \multicolumn{2}{|c|}{ Q3 } & \multicolumn{2}{|c|}{ Q4 } & \\
\hline & & $\mathrm{HR}$ & & $95 \% \mathrm{Cl}$ & $\mathrm{HR}$ & $95 \% \mathrm{Cl}$ & $\mathrm{HR}$ & $95 \% \mathrm{Cl}$ & $\mathrm{HR}$ & $95 \% \mathrm{Cl}$ & \\
\hline \multicolumn{12}{|l|}{ Total legumes } \\
\hline Median intake $(\mathrm{g} / 1000 \mathrm{kcal}(4184 \mathrm{~kJ})) \ddagger$ & 0.0 & \multicolumn{3}{|c|}{$20.7(<30.0)$} & \multirow{2}{*}{\multicolumn{2}{|c|}{$\begin{array}{c}39 \cdot 1(30 \cdot 0,49 \cdot 0) \\
191 / 8838 \cdot 0\end{array}$}} & \multirow{2}{*}{\multicolumn{2}{|c|}{$\begin{array}{cc} & 60.3(49.0,74 \cdot 3) \\
0 & 221 / 8871.0\end{array}$}} & \multicolumn{2}{|c|}{$97.4(\geq 74.3)$} & \\
\hline No. of cases/person-years & $91 / 1941.5$ & \multicolumn{3}{|c|}{$223 / 7934.0$} & & & & & \multicolumn{2}{|c|}{$218 / 8405 \cdot 5$} & \\
\hline Multivariate model $1 \S$ & 1.00 & 0.72 & & $0.56,0.92$ & 0.54 & $0.42,0.69$ & 0.62 & $0.48,0.79$ & 0.64 & $0.50,0.82$ & 0.01 \\
\hline Multivariate model $2 \|$ & 1.00 & 0.68 & & $0.53,0.87$ & 0.47 & $0.37,0.61$ & 0.52 & $0.40,0.67$ & 0.56 & $0.43,0.71$ & $<0.001$ \\
\hline Multivariate model 3ף & 1.00 & $0 \cdot 70$ & & $0.55,0.90$ & 0.48 & $0.37,0.62$ & 0.53 & $0.41,0.67$ & 0.56 & $0.43,0.71$ & $<0.001$ \\
\hline \multicolumn{12}{|l|}{ Dried legumes } \\
\hline Median intake $(\mathrm{g} / 1000 \mathrm{kcal}(4184 \mathrm{~kJ})) \ddagger$ & 0.0 & \multirow{2}{*}{\multicolumn{3}{|c|}{$\begin{array}{l}14.0(<20.5) \\
190 / 7046.0\end{array}$}} & & & \multirow{2}{*}{\multicolumn{2}{|c|}{$\begin{array}{c}40 \cdot 9(33 \cdot 0,51 \cdot 8) \\
176 / 7864 \cdot 0\end{array}$}} & \multirow{2}{*}{\multicolumn{2}{|c|}{$\begin{array}{l}70 \cdot 0(\geq 51 \cdot 8) \\
177 / 7213 \cdot 0\end{array}$}} & \\
\hline No. of cases/person-years & $230 / 6361 \cdot 0$ & & & & & & & & & & \\
\hline Multivariate model 1 & 1.00 & 0.80 & & $0.66,0.97$ & 0.67 & $0.55,0.81$ & 0.66 & $0.54,0.80$ & 0.65 & $0.53,0.79$ & $<0.001$ \\
\hline Multivariate model 2 & 1.00 & 0.74 & & $0.61,0.89$ & 0.59 & $0.48,0.72$ & 0.55 & $0.45,0.68$ & 0.53 & $0.44,0.65$ & $<0.001$ \\
\hline Multivariate model 3 & 1.00 & 0.75 & & $0.61,0.91$ & 0.60 & $0.49,0.73$ & 0.56 & $0.46,0.69$ & 0.53 & $0.43,0.65$ & $<0.001$ \\
\hline \multicolumn{12}{|l|}{ Soyabeans } \\
\hline Median intake $(\mathrm{g} / 1000 \mathrm{kcal}(4184 \mathrm{~kJ})) \ddagger$ & 0.0 & \multicolumn{3}{|c|}{$13.6(<19.5)$} & \multirow{2}{*}{\multicolumn{2}{|c|}{$\begin{array}{c}25 \cdot 2(19 \cdot 5,31 \cdot 2) \\
164 / 7265.5\end{array}$}} & \multirow{2}{*}{\multicolumn{2}{|c|}{$\begin{array}{c}38.9(31.2,49.1) \\
170 / 7577.5\end{array}$}} & \multicolumn{2}{|c|}{$67.4(\geq 49.1)$} & \\
\hline No. of cases/person-years & $270 / 7368 \cdot 0$ & \multicolumn{3}{|c|}{$177 / 6833 \cdot 0$} & & & & & \multicolumn{2}{|c|}{$163 / 6946 \cdot 0$} & \\
\hline Multivariate model 1 & 1.00 & 0.75 & & $0.62,0.91$ & 0.65 & $0.54,0.79$ & 0.65 & $0.53,0.78$ & 0.63 & $0.52,0.76$ & $<0.001$ \\
\hline Multivariate model 2 & 1.00 & 0.71 & & $0.58,0.86$ & 0.57 & $0.47,0.69$ & 0.54 & $0.45,0.66$ & 0.51 & $0.41,0.62$ & $<0.001$ \\
\hline Multivariate model 3 & 1.00 & 0.72 & & $0.59,0.87$ & 0.58 & $0.47,0.71$ & 0.55 & $0.45,0.67$ & 0.51 & $0.41,0.62$ & $<0.001$ \\
\hline \multicolumn{12}{|l|}{ Non-soya beans } \\
\hline Median intake (g/1000 kcal $(4184 \mathrm{~kJ})) \ddagger$ & 0.0 & \multirow{2}{*}{\multicolumn{3}{|c|}{$\begin{array}{l}6.3(<10.8) \\
33 / 1596.0\end{array}$}} & & & & & & & \\
\hline No. of cases/person-years & $802 / 29383.0$ & & & & & & & & & & \\
\hline Multivariate model 1 & 1.00 & 0.76 & & $0.53,1.07$ & 0.79 & $0.57,1 \cdot 10$ & 0.64 & $0.44,0.93$ & 0.85 & $0.63,1.16$ & 0.01 \\
\hline Multivariate model 2 & 1.00 & 0.68 & & $0.48,0.96$ & 0.73 & $0.53,1.02$ & 0.63 & $0.43,0.91$ & 0.92 & $0.67,1.25$ & 0.01 \\
\hline Multivariate model 3 & 1.00 & 0.69 & & $0.49,0.98$ & 0.75 & $0.54,1.04$ & 0.63 & $0.43,0.91$ & 0.88 & $0.65,1.20$ & 0.01 \\
\hline Fresh legumes & & & & & & & & & & & \\
\hline Median intake $(\mathrm{g} / 1000 \mathrm{kcal}(4184 \mathrm{~kJ})) \ddagger$ & 0.0 & & $14.8(<20.9)$ & & & & & & & & \\
\hline No. of cases/person-years & $333 / 8039.5$ & & $147 / 6957 \cdot 0$ & & & & & & & & \\
\hline Multivariate model 1 & 1.00 & 0.57 & & $0.47,0.69$ & 0.52 & $0.42,0.63$ & 0.56 & $0.46,0.68$ & 0.64 & $0.53,0.77$ & $<0.001$ \\
\hline Multivariate model 2 & 1.00 & 0.55 & & $0.45,0.67$ & 0.48 & $0.39,0.59$ & 0.52 & $0.43,0.63$ & 0.66 & $0.55,0.79$ & $<0.001$ \\
\hline Multivariate model 3 & 1.00 & 0.56 & & $0.46,0.68$ & 0.49 & $0.40,0.60$ & 0.53 & $0.44,0.64$ & 0.67 & $0.55,0.81$ & $<0.001$ \\
\hline
\end{tabular}

Q, quartile; ref, reference group.

The non-consumers were set as the reference in all statistical models.

$\dagger P_{\text {for trend }}$ values were calculated across the categories of legume intakes by dealing each ordinal score variable as a continuous variable in the model.

$\mp$ Median intake (range in parentheses).

Covariates: age (continuous) and sex

॥ Covariates: included the variables listed in footnote § plus BMI (continuous), physical activity (tertiles of metabolic equivalents), education ( $\leq 6,7-9$, or $\geq 10$ years), per capital household income (tertiles), urbanisation index (tertiles), smoking (never/ever), alcohol drinking (never/ever), diabetes (yes/no).

ๆ Covariates: further added dietary intakes (continuous) of total energy (kcal/d), total fruits and vegetables ( $\mathrm{g} / 1000 \mathrm{kcal}(4184 \mathrm{~kJ})$ ), as well as total red meat ( $\mathrm{g} / 1000 \mathrm{kcal}(4184 \mathrm{~kJ})$ ) into the model in addition to variables listed in footnote II. 
Table 3. Risk* of incident hypertension by categories of cumulative average total legume intake stratified by various characteristics of participants in the China Nutrition and Health Study (CHNS) 2004-2011

(Multivariate hazard ratios (HR) and $95 \%$ confidence intervals)

\begin{tabular}{|c|c|c|c|c|c|c|c|c|c|c|c|}
\hline & \multicolumn{9}{|c|}{ Levels of total legume intake } & \multirow[b]{3}{*}{$P_{\text {for trend }} \ddagger$} & \multirow[b]{3}{*}{$P_{\text {for interaction }} \S$} \\
\hline & \multirow{2}{*}{$\begin{array}{c}\text { Non- } \\
\text { consumers } \\
\text { (ref) } \dagger\end{array}$} & \multicolumn{2}{|r|}{ Q1 } & \multicolumn{2}{|r|}{ Q2 } & \multicolumn{2}{|r|}{ Q3 } & \multicolumn{2}{|r|}{ Q4 } & & \\
\hline & & $\mathrm{HR}$ & $95 \% \mathrm{Cl}$ & $\mathrm{HR}$ & $95 \% \mathrm{Cl}$ & $\mathrm{HR}$ & $95 \% \mathrm{Cl}$ & $\mathrm{HR}$ & $95 \% \mathrm{Cl}$ & & \\
\hline \multicolumn{12}{|l|}{ Stratified by sex\|l| } \\
\hline Male & 1.00 & 0.60 & $0.42,0.85$ & 0.40 & $0.28,0.58$ & 0.44 & $0.31,0.63$ & 0.44 & $0.31,0.63$ & $<0.001$ & 0.14 \\
\hline Female & 1.00 & 0.81 & $0.57,1.16$ & 0.57 & $0.39,0.82$ & 0.61 & $0.43,0.87$ & 0.68 & $0.48,0.97$ & 0.04 & \\
\hline \multicolumn{12}{|l|}{ Stratified by age } \\
\hline Age $<65$ years & 1.00 & 0.67 & $0.49,0.91$ & 0.48 & $0.35,0.66$ & 0.57 & $0.42,0.78$ & 0.61 & $0.45,0.83$ & 0.04 & 0.02 \\
\hline Age $\geq 65$ years & 1.00 & 0.73 & $0.48,1 \cdot 12$ & 0.55 & $0.35,0.85$ & 0.43 & $0.28,0.68$ & 0.47 & $0.30,0.73$ & $<0.001$ & \\
\hline \multicolumn{12}{|l|}{ Stratified by BMI } \\
\hline $\mathrm{BMl}<24 \mathrm{~kg} / \mathrm{m}^{2}$ & 1.00 & 0.66 & $0.47,0.94$ & 0.53 & $0.37,0.75$ & 0.56 & $0.39,0.79$ & 0.64 & $0.46,0.91$ & 0.06 & 0.10 \\
\hline $\mathrm{BMI} \geq 24 \mathrm{~kg} / \mathrm{m}^{2}$ & 1.00 & 0.75 & $0.52,1.08$ & 0.48 & $0.33,0.69$ & 0.52 & $0.36,0.75$ & 0.52 & $0.36,0.74$ & $<0.001$ & \\
\hline \multicolumn{12}{|l|}{ Stratified by physical activity } \\
\hline Physical activity < median level & 1.00 & 0.76 & $0.56,1.03$ & 0.54 & $0.40,0.74$ & 0.51 & $0.38,0.70$ & 0.57 & $0.42,0.77$ & $<0.001$ & 0.28 \\
\hline Physical activity $\geq$ median level & 1.00 & 0.61 & $0.39,0.95$ & 0.39 & $0.25,0.61$ & 0.56 & $0.36,0.86$ & 0.54 & $0.35,0.84$ & $0 \cdot 14$ & \\
\hline \multicolumn{12}{|l|}{ Stratified by cigarette smoking } \\
\hline Never & 1.00 & 0.76 & $0.57,1.03$ & 0.55 & $0.41,0.75$ & 0.52 & $0.38,0.70$ & 0.58 & $0.43,0.78$ & $<0.001$ & 0.34 \\
\hline Ever & 1.00 & 0.54 & $0.35,0.85$ & 0.36 & $0.22,0.57$ & 0.51 & $0.33,0.80$ & 0.50 & $0.31,0.78$ & $0 \cdot 12$ & \\
\hline \multicolumn{12}{|l|}{ Stratified by alcohol drinking } \\
\hline Never & 1.00 & 0.83 & $0.61,1.12$ & 0.52 & $0.38,0.71$ & 0.60 & $0.44,0.81$ & 0.66 & $0.49,0.89$ & 0.01 & 0.12 \\
\hline Ever & 1.00 & 0.46 & $0.30,0.72$ & 0.39 & $0.25,0.60$ & 0.38 & $0.24,0.59$ & 0.37 & $0.23,0.58$ & 0.001 & \\
\hline
\end{tabular}

ref, Reference group; Q, quartile.

* Covariates: age (continuous), sex, BMI (continuous), physical activity (tertiles of metabolic equivalents), education ( $\leq 6,7-9$, or $\geq 10$ years), per capital household income (tertiles), urbanisation index (tertiles), smoking (never/ever), alcohol drinking (never/ever), diabetes (yes/no), dietary intakes (continuous) of total energy (kcal ${ }^{\star \star} / \mathrm{d}$ ), total fruits and vegetables $(\mathrm{g} / 1000 \mathrm{kcal}(4184 \mathrm{~kJ}))$, as well as total red meat $(\mathrm{g} / 1000 \mathrm{kcal}(4184 \mathrm{~kJ}))$, except for the stratifying variables per se.

$\dagger$ The non-consumers were set as the reference in all statistical models.

$\ddagger P_{\text {for trend }}$ values were calculated across the categories of total legume intake by dealing each ordinal score variable as a continuous variable in the model.

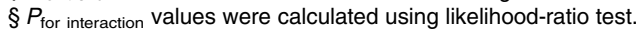

II In the stratified analysis, characteristics of participants at baseline were used for stratification and adjustment.

${ }^{\star \star}$ To convert kcal to kJ, multiply by $4 \cdot 184$.

\section{Discussion}

In this cohort of Chinese general population, we found that higher intakes of total legumes, fresh legumes and soyabeans but not non-soyabeans, were strongly associated with a reduced risk of developing hypertension. These associations were independent of established risk factors for hypertension, as well as other major dietary confounders, and largely persisted in subgroup analyses.

Prior epidemiological studies of the association between legume intake and hypertension risk were inconsistent. Papanikolaou and Fulgoni III utilising data from National Health and Examination Survey (NHANES 1999-2002) found that bean consumers had lower systolic BP and a reduced risk of elevated systolic BP in comparison with non-consumers ${ }^{(8)}$, which confirmed our results that the risk of hypertension could be expectably reduced in virtue of regular legume consumption. However, these findings are discordant with those of Golzarand et al. who reported no significant relationship between legumes and development of hypertension over 3 years ${ }^{(16)}$. Importantly, compared with daily average legume intake at $52.5 \mathrm{~g} / 1000 \mathrm{kcal}$ $(4184 \mathrm{~kJ})$ in our cohort, the highest legume intake $(41.9 \mathrm{~g} / \mathrm{d})$ in this Iran cohort may have not been enough to produce significant effects on hypertension endpoints during a short follow-up period. Besides, our study was consistent with some but not all clinical trials $^{(10,13,14)}$. For example, a small-scale 6-week randomised trial concluded that substitution of red meat with legume intake had no significant effect on systolic or diastolic $\mathrm{BP}^{(13)}$.
Such inconsistencies may be partly attributed to the differences in study populations, design and measures of outcome. Also, short-run intervention cannot rule out the long-term benefits from consuming legumes with regard to hypertension prevention, as found in our study.

The mechanisms through which legume consumption protects against hypertension have been suggested. Dietary fibre, plant protein and unsaturated fat are all commonly found in legumes and are inversely related to BP in previous studies $^{(34-36)}$. The possible biological pathways may involve energy regulation, serum lipids improvement, blood glucose control, as well as weight loss, all of which could help reduce hypertension risk $^{(12,37)}$. In addition, legumes contain large amounts of minerals such as $\mathrm{K}, \mathrm{Ca}$ and $\mathrm{Mg}$, which can reduce peripheral vascular resistance by virtue of facilitating the synthesis of vasodilator prostacyclin and $\mathrm{NO}$, as well as lowering angiotensin II levels ${ }^{(38,39)}$. $\mathrm{K}$ is also involved in the modulation of extracellular fluid volume and accordingly helps regulate arterial pressure $^{(40)}$. Moreover, legumes have other nutrients such as folic acid that lower plasma homocysteine levels to reduce the hypertension risk $^{(37)}$. Overall, the possible beneficial effects of total legumes on hypertension development have a strong biological basis owing to their various protective nutrient constituents.

In subgroup analysis, we identified that intakes of fresh legumes and soyabeans rather than non-soyabeans were prominently associated with lower hypertension risks, which is in part 
consistent with some previous research that has reported associations for specific legume types. Longitudinal study conducted in America by Borgi et al. have shown a significant decrease in hypertension risk with intake of tofu and soyabeans but not other beans ${ }^{(41)}$. Yang et al. also found that usual intake of soya foods was associated with reduction of both systolic and diastolic $\mathrm{BP}$ among late postmenopausal women ${ }^{(17)}$. It is plausible that different legumes packed with varying nutrients may exert discrepant effects on $\mathrm{BP}^{(18,19)}$. For soyabeans, their higher content of soya protein is reported to have markedly high biological value especially pertaining to reducing BP among prehypertensive patients ${ }^{(42)}$ and the potential pathways involve increasing NO levels ${ }^{(43)}$, inhibiting angiotensin-converting enzyme and producing a natriuretic effect similar to furosemide ${ }^{(44,45)}$. Also, soya isoflavones acting as oestrogen mimics have gained high credit for deceasing in vivo oxidation, facilitating NO-dependent endothelial vasodilation and thereby relieving systemic arterial stiffness ${ }^{(46-48)}$. Fresh legumes (such as string beans), which is also referred to as 'leguminous vegetables', could deliver unique nutritional benefits associated with not only legumes but also vegetables; their antioxidant contents including vitamin $\mathrm{C}$ and carotenoids like lutein may well contribute to their possible hypotensive health effects ${ }^{(49)}$. Of note, fresh legumes were commonly consumed at high levels ( $29 \cdot 7 \mathrm{~g} / 1000 \mathrm{kcal}(4184 \mathrm{~kJ})$ per d) among our participants, which renders their potential protect role against hypertension development. Regarding the null results for non-soyabeans, it may attribute to the limited consumption rate $(15.8 \%)$ or low average intake level in the present study or a lack of true association with hypertension. More data are needed to clarify the underlying nutritional links.

Several strengths enhanced the validity of our study, including its large sample size, repeated assessments of exposure, multivariate adjustment and the hierarchical evaluation of the relationships between various legume consumption and hypertension risk. Besides, the prospective study design helped to eliminate potential recall bias which is of particular concern in most retrospective post hoc analysis. Limitations of our study also merit consideration. First, residual or unmeasured confounders cannot be completely ruled out and thus hinder causal inference based on these observations; however, we carefully controlled for a broad range of potential covariates, and the stratification analyses as well as sensitivity analyses (such as further adjustment for $\mathrm{Na}$ intake) did not appreciably alter our results. Second, even though the dietary assessment has been previously validated $^{(26,27)}$ and a large proportion of subjects reported acceptable EI, misreporting of dietary information is an unavoidable concern in the process of three consecutive 24-h food recalls. Nevertheless, due to the prospective study design, potential misclassification of legume intakes was unlikely to depend on study outcome and hence prone to biasing true associations toward the null. Third, we adopted uncooked (dried) weight to measure the intake of dried legumes which might lead to measurement errors, although traditional cooking in China would seldom alter the profile of legumes. Fourth, the definition of hypertension was not validated and based largely (approximately $99.7 \%$ ) on self-reported diagnoses from physicians. Although self-report of hypertension diagnosis has been suggested as a relatively reliable tool in previous research ${ }^{(50)}$, it could still inevitably introduce potential misclassification bias and dilute the association. Also, we were not able to cover the variable on receiving antihypertensive drugs due to substantial missingness, which might have influenced the sensitivity of defining hypertension cases. Fifth, participants lost to follow-up in the present study were generally younger with less vigorous exercises and at higher socio-economic levels. Nevertheless, their legume intake and other important known risk factors for hypertension (such as BMI and smoking) did not vary from the included subjects, which makes our findings not much biased. Last, the participants included in the present study were predominantly Chinese adults with Asian ancestry, and it is unsure whether our findings could be generalised to other ethnic groups, so the results should be interpreted with caution.

In conclusion, the results of this cohort study demonstrated that usual intake of total legumes, fresh legumes, soyabeans except non-soyabeans, could act as a potential protective factor for hypertension incidence. According to our present knowledge and dietary recommendations to increase fruit and vegetable consumption, increasing intake of legumes may be an important part of diet interventions to reduce hypertension risk. Public health initiatives on the primary prevention of hypertension may consider our results as a rational and feasible dietary modification, with support from further appropriately designed intervention studies and formative research.

\section{Acknowledgements}

The present study used data from China Health and Nutrition Survey (CHNS). We thank the National Institute for Nutrition and Health, Chinese Center for Disease Control and Prevention, Carolina Population Center, the University of North Carolina at Chapel Hill, the National Institutes of Health (NIH), and the Fogarty International Center, NIH for financial support for the collection and analysis files of the CHNS data from 1989 to 2011 and the future surveys, and the China-Japan Friendship Hospital, Ministry of Health for support for CHNS 2009, Chinese National Human Genome Center at Shanghai since 2009 and Beijing Municipal Center for Disease Prevention and Control since 2011.

This research was funded partly by grants from the New-star Plan of Science and Technology of Shaanxi Province (2015LJXX-07), the China Postdoctoral Science Special Foundation (2015T81036) and the Fundamental Research Funds for the Central Universities (qngz2016004). The funders had no role in the study design, implementation, analysis, decision to publish or reparation of the manuscript.

The authors' contributions were as follows: F. G. and L. M. conceived and designed the study. F. G. and Q. Z. conducted the research, including the literature review, statistical analyses and results interpretation. F. G., Q. Z. and L. M. were involved in the manuscript writing. Y. Y., Y. L., N. Y., J. L. and X. H. L. revised the manuscript. All the authors read and approved the final manuscript.

The authors declare no conflicts of interest. 


\section{Supplementary material}

For supplementary material referred to in this article, please visit https://doi.org/10.1017/S0007114519002812

\section{References}

1. Healthdata (2016) Global burden of disease arrow diagram. http://www.healthdata.org/gbd/data-visualizations (accessed November 2018).

2. Mills KT, Bundy JD, Kelly TN, et al. (2016) Global disparities of hypertension prevalence and controlclinical perspective: a systematic analysis of population-based studies from 90 countries. Circulation 134, 441-450.

3. Lu J, Lu Y, Wang X, et al. (2017) Prevalence, awareness, treatment, and control of hypertension in China: data from 1.7 million adults in a population-based screening study (China PEACE Million Persons Project). Lancet 390, 2549-2558.

4. Sacks FM, Svetkey LP, Vollmer WM, et al. (2001) Effects on blood pressure of reduced dietary sodium and the Dietary Approaches to Stop Hypertension (DASH) diet. N Engl J Med 344, 3-10.

5. Blumenthal JA, Babyak MA, Hinderliter A, et al. (2010) Effects of the DASH diet alone and in combination with exercise and weight loss on blood pressure and cardiovascular biomarkers in men and women with high blood pressure: the ENCORE study. Arch Intern Med 170, 126-135.

6. Bouchenak M \& Lamri-Senhadji M (2013) Nutritional quality of legumes, and their role in cardiometabolic risk prevention: a review. J Med Food 16, 185-198.

7. Martínez R, López-Jurado M, Wanden-Berghe C, et al. (2016) Beneficial effects of legumes on parameters of the metabolic syndrome: a systematic review of trials in animal models. Br J Nutr 116, 402-424.

8. Papanikolaou Y \& Fulgoni III VL (2008) Bean consumption is associated with greater nutrient intake, reduced systolic blood pressure, lower body weight, and a smaller waist circumference in adults: results from the National Health and Nutrition Examination Survey 1999-2002. J Am Coll Nutr 27, 569-576.

9. Hermsdorff HHM, Zulet MA, Abete I, et al. (2011) A legumebased hypocaloric diet reduces proinflammatory status and improves metabolic features in overweight/obese subjects. Eur J Nutr 50, 61-69.

10. Hosseinpour-Niazi S, Mirmiran P, Hedayati M, et al. (2015) Substitution of red meat with legumes in the therapeutic lifestyle change diet based on dietary advice improves cardiometabolic risk factors in overweight type 2 diabetes patients: a cross-over randomized clinical trial. Eur J Clin Nutr 69 , 592-597.

11. Hosseinpour-Niazi S, Mirmiran P, Amiri Z, et al. (2012) Legume intake is inversely associated with metabolic syndrome in adults. Arch Iran Med 15, 538-544.

12. Jenkins DJ, Kendall CW, Augustin LS, et al. (2012) Effect of legumes as part of a low glycemic index diet on glycemic control and cardiovascular risk factors in type 2 diabetes mellitus: a randomized controlled trial. Arch Intern Med 172, 1653-1660.

13. Saraf-Bank S, Esmaillzadeh A, Faghihimani E, et al. (2016) Effects of legume-enriched diet on cardiometabolic risk factors among individuals at risk for diabetes: a crossover study. J Am Coll Nutr 35, 31-40.

14. Jayalath VH, De Souza RJ, Sievenpiper JL, et al. (2013) Effect of dietary pulses on blood pressure: a systematic review and meta-analysis of controlled feeding trials. Am J Hypertens 27, 56-64.
15. Weng LC, Steffen LM, Szklo M, et al. (2013) A diet pattern with more dairy and nuts, but less meat is related to lower risk of developing hypertension in middle-aged adults: the Atherosclerosis Risk in Communities (ARIC) study. Nutrients 5, 1719-1733.

16. Golzarand M, Bahadoran Z, Mirmiran P, et al. (2016) Protein foods group and 3-year incidence of hypertension: a prospective study from Tehran Lipid and Glucose Study. J Renal Nutr 26, 219-225.

17. Yang G, Shu XO, Jin F, et al. (2005) Longitudinal study of soy food intake and blood pressure among middle-aged and elderly Chinese women. Am J Clin Nutr 81, 1012-1017.

18. Ren SC, Liu ZL \& Wang P (2012) Proximate composition and flavonoids content and in vitro antioxidant activity of 10 varieties of legume seeds grown in China. $J$ Med Plants Res 6, 301-308.

19. Nozue M, Shimazu T, Sasazuki S, et al. (2017) Fermented soy product intake is inversely associated with the development of high blood pressure: The Japan Public Health Center-Based Prospective Study. J Nutr 147, 1749-1756.

20. Li L, Yang T, Liu R, et al. (2017) Food legume production in China. Crop J 5, 115-126.

21. Rizzo G \& Baroni L (2018) Soy, soy foods and their role in vegetarian diets. Nutrients 10, 43-93.

22. Popkin BM, Du S, Zhai F, et al. (2009) Cohort Profile: The China Health and Nutrition Survey-monitoring and understanding socio-economic and health change in China, 1989-2011. Int J Epidemiol 39, 1435-1440.

23. Du S, Neiman A, Batis C, et al. (2013) Understanding the patterns and trends of sodium intake, potassium intake, and sodium to potassium ratio and their effect on hypertension in China. Am J Clin Nutr 99, 334-343.

24. Huang CC, Yabiku ST \& Kronenfeld JJ (2015) The effects of household technology on body mass index among Chinese adults. Pop Res Pol Rev 34, 877-899.

25. Wang Z, Zhang B, Wang H, et al. (2013) Study on the multilevel and longitudinal association between red meat consumption and changes in body mass index, body weight and risk of incident overweight among Chinese adults. Chin J Epidemiol 34, 661-667.

26. Yao M, McCrory MA, Ma G, et al. (2003) Relative influence of diet and physical activity on body composition in urban Chinese adults. Am J Clin Nutr 77, 1409-1416.

27. Li Y, He Y, Zhai F, et al. (2006) Comparison of assessment of food intakes by using 3 dietary survey methods. Clin J Prev Med 40, 273-280.

28. Institute of Medicine (2005) Energy. In Dietary Reference Intakes for Energy, Carbohydrate, Fiber, Fat, Fatty Acids, Cholesterol, Protein, and Amino Acids, pp. 107-264 [GE Spears, editor]. Washington, DC: National Academies Press.

29. Black AE \& Cole TJ (2000) Within- and between-subject variation in energy expenditure measured by the doubly-labelled water technique: implications for validating reported dietary energy intake. Eur J Clin Nutr 54, 386-394.

30. Nelson M, Black AE, Morris JA, et al. (1989) Between- and within-subject variation in nutrient intake from infancy to old age: estimating the number of days required to rank dietary intake with desired precision. Am J Clin Nutr 50, 156-167.

31. Willett W, Stampfer MJ (2013) Implications of total energy intake for epidemiologic analysis. In Nutritional Epidemiology [W Willett, editor]. Oxford: Oxford University Press, p. 260.

32. Ainsworth BE, Haskell WL, Herrmann SD, et al. (2011) 2011 Compendium of Physical Activities: a second update of codes and MET values. Med Sci Sports Exerc 43, $1575-1581$. 
33. Vatcheva KP, Lee M, McCormick JB, et al. (2016) Multicollinearity in regression analyses conducted in epidemiologic studies. Epidemiology (Sunnyvale) 6, 1-9.

34. Streppel MT, Arends LR, van't Veer P, et al. (2005) Dietary fiber and blood pressure: a meta-analysis of randomized placebocontrolled trials. Arch Intern Med 165, 150-156.

35. Tielemans SM, Altorf-van der Kuil W, Engberink MF, et al. (2013) Intake of total protein, plant protein and animal protein in relation to blood pressure: a meta-analysis of observational and intervention studies. J Hum Hypertens 27, 564-571.

36. Yang B, Shi MQ, Li ZH, et al. (2016) Fish, long-chain $n-3$ PUFA and incidence of elevated blood pressure: a meta-analysis of prospective cohort studies. Nutrients $8,58-69$.

37. Rebello C, Greenway F \& Finley JW (2014) A review of the nutritional value of legumes and their effects on obesity and its related co-morbidities. Obes Rev 15, 392-407.

38. Adrogué HJ \& Madias NE (2007) Sodium and potassium in the pathogenesis of hypertension. N Engl J Med 356, 1966-1978.

39. Schutten JC, Joosten MM, de Borst MH, et al. (2018) Magnesium and blood pressure: a physiology-based approach. Adv Chronic Kidney Dis 25, 244-250.

40. Terker AS, Zhang C, McCormick JA, et al. (2015) Potassium modulates electrolyte balance and blood pressure through effects on distal cell voltage and chloride. Cell Metab 21, 39-50.

41. Borgi L, Muraki I, Satija A, et al. (2015) Fruit and vegetable consumption and the incidence of hypertension in three prospective cohort studies. Hypertension 67, 288-293.

42. Kwak JH, Kim M, Lee E, et al. (2013) Effects of black soy peptide supplementation on blood pressure and oxidative stress: a randomized controlled trial. Hypertens Res 36, 1060-1066.
43. Kelm M (2003) The L-arginine-nitric oxide pathway in hypertension. Curr Hypertens Rep 5, 80-86.

44. Yang HY, Yang SC, Chen JR, et al. (2004) Soyabean protein hydrolysate prevents the development of hypertension in spontaneously hypertensive rats. Br J Nutr $\mathbf{9 2}, 507-512$.

45. He J, Gu D, Wu X, et al. (2005) Effect of soybean protein on blood pressure: a randomized, controlled trial. Ann Intern Med 143, 1-9.

46. Valsecchi AE, Franchi S, Panerai AE, et al. (2011) The soy isoflavone genistein reverses oxidative and inflammatory state, neuropathic pain, neurotrophic and vasculature deficits in diabetes mouse model. Eur J Pharmacol 650, 694-702.

47. Si H \& Liu D (2008) Genistein, a soy phytoestrogen, upregulates the expression of human endothelial nitric oxide synthase and lowers blood pressure in spontaneously hypertensive rats. J Nutr 138, 297-304.

48. Hazim S, Curtis PJ, Schär MY, et al. (2016) Acute benefits of the microbial-derived isoflavone metabolite equol on arterial stiffness in men prospectively recruited according to equol producer phenotype: a double-blind randomized controlled trial. Am J Clin Nutr 103, 694-702.

49. Baardseth P, Bjerke F, Martinsen BK, et al. (2010) Vitamin C, total phenolics and antioxidative activity in tip-cut green beans (Phaseolus vulgaris) and swede rods (Brassica napus var. napobrassica) processed by methods used in catering. $J$ Sci Food Agric 90, 1245-1255.

50. Ahn SN, Zhao H, Smith ML, et al. (2011) BMI and lifestyle changes as correlates to changes in self-reported diagnosis of hypertension among older Chinese adults. I Am Soc Hypertens 5, 21-30 